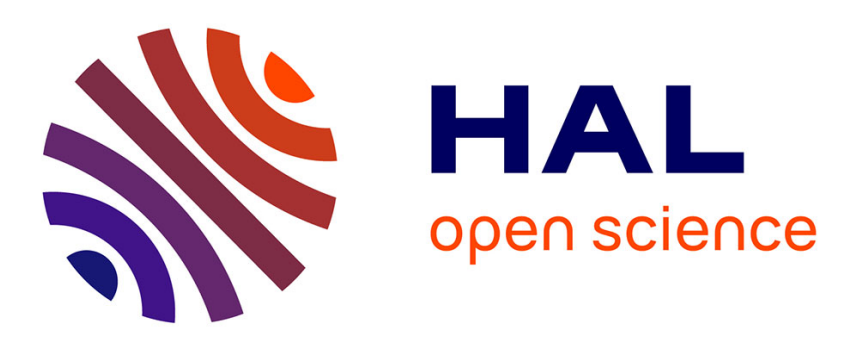

\title{
Introduction à la théorie analytique de la traduction et de l'interprétation
}

\author{
Mathieu Guidère
}

\section{To cite this version:}

Mathieu Guidère. Introduction à la théorie analytique de la traduction et de l'interprétation. Babel, 2010, 56 (4), pp.299-312. hal-00945743

\section{HAL Id: hal-00945743 \\ https://hal.science/hal-00945743}

Submitted on 12 Feb 2014

HAL is a multi-disciplinary open access archive for the deposit and dissemination of scientific research documents, whether they are published or not. The documents may come from teaching and research institutions in France or abroad, or from public or private research centers.
L'archive ouverte pluridisciplinaire HAL, est destinée au dépôt et à la diffusion de documents scientifiques de niveau recherche, publiés ou non, émanant des établissements d'enseignement et de recherche français ou étrangers, des laboratoires publics ou privés. 


\title{
Introduction à la théorie analytique de la traduction et de l'interprétation
}

\author{
Mathieu Guidère \\ Université de Genève
}

Dans Pour la poétique II (1973), Henri Meschonnic insiste sur l'importance de l'idéologie dans l'étude de la traduction: «La théorie de la traduction des textes se situe dans le travail, fondamental pour l'épistémologie, sur les rapports entre pratique empirique et pratique théorique, écriture et idéologie, science et idéologie (.. .) Une théorie translinguistique de l'énonciation consiste dans l'interaction entre une linguistique de l'énonciation [. . . et une théorie de l'idéologie» (Meschonnic 1973: 305).

Pour lui, la notion de "transparence» de la traduction reflète simplement l'ignorance du traducteur, car la traduction n'est autre chose que la «ré-énonciation spécifique d'un sujet historique» (proposition 11): "L'illusion de la transparence appartient au système idéologique caractérisé par les notions liées d'hétérogénéité entre la pensée et le langage» (Meschonnic 1973: 305).

Pour justifier cette position, Meschonnic insiste sur le lien indéfectible dans le cadre de la traduction entre écriture et idéologie: "une théorie et une pédagogie des textes, désesthétisés, désacralisés, travaillant à une sémantique théorique du langage poétique et aux rapports entre écriture et idéologie, peut transformer le statut théorique, la pratique et le statut sociologique de la traduction» (Meschonnic 1973 : 323).

Dans L'épreuve de l'étranger (1984), Antoine Berman estime que la réflexion sur la traduction est devenue une nécessité interne. La question éthique est intiment liée au «drame du traducteur», tiraillé entre deux pôles, l'œuvre et l'auteur, l'auteur et le public: "Traduire, c'est servir deux maîtres»(Rosenzweig). La réflexion est également indispensable parce que les cultures résistent à la traduction - même si elles en ont besoin - par pur réflexe ethnocentrique. Or, l'essence de la traduction est d'être ouverture, dialogue, métissage, décentrement. Cette éthique "positive» s'oppose, selon Berman, à une éthique «négative» qui cherche à détourner la traduction de sa visée humaniste en la mettant au service de valeurs idéologiques qui opèrent une négation de «l'étrangeté» de l'Autre ou de l'œuvre traduite. C'est pourquoi, le traducteur doit développer une «analytique» lui permettant de

Babel 56:4 (2010), 1-14. C Fédération des Traducteurs (FIT) Revue Babel DOI 10.1075/babel.56.4.oxgui ISSN 0521-9744 E-ISSN 1569-9668 
repérer les systèmes de déformation qui menacent ses pratiques et opèrent de façon consciente ou inconsciente au niveau de ses choix de traduction. Bref, la traduction-analyse s'impose au traducteur pour contrôler sa "pulsion traductrice».

Dans Éthique et politique du traduire (2007), Meschonnic développe trois concepts (poétique, éthique et politique) qui participent d'une théorie d'ensemble du langage dans laquelle la traduction joue un rôle déterminant:

je ne définis pas l'éthique comme une responsabilité sociale, mais comme la recherche d'un sujet qui s'efforce de se constituer comme sujet par son activité, mais une activité telle qu'est sujet celui par qui un autre est sujet. Et en ce sens, comme être de langage, ce sujet est inséparablement éthique et poétique. C'est dans la mesure de cette solidarité que l'éthique du langage concerne tous les êtres de langage, citoyens de l'humanité, et c'est en quoi l'éthique est politique (Meschonnic 2007: 8).

\section{Les fondements de la traduction-analyse}

Le premier élément de fond conduisant à ce nouveau paradigme est le phénomène de politisation accrue du langage. Celui-ci s'affirme de plus en plus comme un outil idéologique et comme un enjeu politique de premier plan, deux aspects dont il n'est plus possible de faire abstraction dans la plupart des régions du monde.

Le second élément est celui d'une connaissance de plus en plus fine des processus cognitifs mis en œuvre par l'humain dans la réalisation d'une traduction, et cela quelle que soit la nature du texte-à-traduire. Même «l'intuition" du traducteur est de mieux en mieux connue et analysée de façon scientifique dans le cadre des sciences cognitives. L'interdisciplinarité a donc permis d'appréhender la complexité d'un métier que l'on croyait insondable.

Ces éléments possèdent des tenants et des aboutissants qui méritent d'être expliqués en détail parce qu'ils induisent une nouvelle conception de la traduction et une vision renouvelée du traducteur.

Cette conception est articulée autour du concept de « réflexivité» qui englobe à la fois la réflexion du traducteur sur son travail et le caractère réflexif du processus de traduction. Selon les cas, cette réflexivité peut apparaître comme un miroir de l'état d'esprit du traducteur ou un prisme déformant de ses conceptions, perceptions et intentions.

La traduction-analyse récuse plusieurs postulats de la traductologie classique:

1. Elle refuse la séparation du texte-à-traduire et du sujet-traduisant, car les deux sont indissociables dans l'activité de traduction: l'un fait exister à l'autre et l'actualise.

2. Elle récuse le fait que le «sens» soit dans le texte: il est un devenir tributaire de la compétence du traducteur, qui l'actualise que ce soit au départ ou à l'arrivée du processus. 
3. Elle refuse le caractère énigmatique du «sens» et le considère comme une «orientation» de l'esprit du traducteur à un moment donné concernant un sujet particulier. Interpréter n'est pas traduire: l'interprétation elle-même est tributaire de la compréhension du traducteur.

4. Elle conteste la fonctionnalisation du texte-à-traduire parce que la fonction ou le skopos sont eux-mêmes des données subjectives, changeantes et dépendants du traducteur. La finalité de l'acte de traduire elle-même est évolutive et circonstancielle; elle ne peut être prise comme fondement à la traduction.

5. Sur le fond, la traduction-analyse vise l'autonomisation de la traductologie et s'oppose, par conséquent, à une vision aliénante de la traduction comme relevant des paradigmes de l'information ou de la communication (émetteur, récepteur, etc.).

\section{Le sens est orienté}

On sait depuis longtemps que le langage possède une «praxis» intrinsèque et que les traducteurs ne peuvent ignorer cette propriété langagière. Et pourtant, les traducteurs continuent de traduire comme si le langage était transparent, comme s'il était une succession de vocables spécialisés et de notions aseptisés. Que ce soit par ignorance ou par inconscience, le produit final de ces traductions parait faussé alors même qu' il se veut «fidèle» à l'original.

La traduction n'est pas une activité «neutre» signifie que le sujet traduisant véhicule, malgré lui, un ensemble de vocables et d'expressions dont il n'est pas possible de réduire la charge idéologique, émotionnelle ou proprement politique. C'est le cas de l'écrasante majorité des mots de la langue, y compris dans les domaines dits scientifiques ou techniques. Or, l'on constate souvent que le traducteur neutralise cette charge afin de pouvoir transmettre le sens objectivé dans une autre langue.

Certes, il peut y avoir perte de sens lors de la traduction, mais lorsque l'essentiel réside justement dans la charge signifiante neutralisée, la question de la validité de la traduction devient centrale. Dans la pratique, non seulement les mots à traduire ne sont pas neutres, mais en plus le langage est chargé d'une intentionnalité évidente.

Il faut distinguer ici entre l'«intention» et l'«intentionnalité». La première renvoie à une conception de la communication humaine envisagée comme volontaire et consciente, ce qui n'est pas toujours le cas. La seconde (l'intentionnalité) est de nature phénoménologique et subsume les notions de finalité et de motivation. Elle permet de concevoir la traduction comme un acte téléologique mû par une visée réfléchie. 
Ainsi, traduire un texte d'Al-Qaïda implique, avant tout, une prise de conscience du fait que les mots eux-mêmes sont chargés d'une intentionnalité propre. Mais la traduction neutralisante devient, par là même, intentionnelle, en ce sens que le commanditaire et/ou le traducteur possèdent une visée propre qui sous-tend leur travail et qui détermine jusqu'aux choix des équivalences entre les langues.

Dans Parcours de la reconnaissance, le philosophe Paul Ricœur part de l'analyse sémantique du verbe «reconnaître» pour construire sa théorie de la connaissance. Il explique ainsi le déplacement de sens à partir de l'idée de «reconnaître»: "se reconnaître soi-même», "se reconnaître réciproquement», "être reconnu», etc. Son objectif est de mettre en évidence les constructions mentales qui se profilent derrière les mots afin de prendre en considération le poids de la culture collective dans le remodelage de la langue.

Le texte-à-traduire est protéiforme et les choix de traduction souvent multiples. Dès lors, qu'est-ce qui va attirer l'attention du traducteur? Et à quelles caractéristiques sera-t-il attaché dans sa traduction? Car à y regarder de près, la perception du traducteur est non seulement porteuse d'interprétation mais elle véhicule aussi des préjugés et de fausses idées qui s'immiscent parfois dans les interstices de la traduction. Certes, le traducteur transmet bien une certaine vérité, certes son intuition et son expérience permettent de saisir le sens du texte, mais sa perception est porteuse d'un choix personnel et subjectif concernant ce que dit précisément le texte. Même lorsque sa perception ne s'accompagne pas de réflexion sur le signification véritable du texte, elle lui donne sens et décide de ce qu'il veut dire pour le récepteur final qui n’a pas accès à l'original.

Cette opération est d'autant plus cruciale lorsque le texte est ambigu et qu'il est susceptible de recevoir plus d'une interprétation. La traduction ne nous met pas directement au contact du texte mais des mots choisis par le traducteur du texte. L'objet auquel elle nous donne accès n'est pas le texte lui-même mais une certaine compréhension de ce texte. Le sens transmis au récepteur final, en passant par le biais de la traduction, paraît être toujours «orienté» ou du moins «subjectivé ».

Deux problèmes majeurs peuvent dès lors être formulés, mais qui n'épuisent pas les questions posées par la traduction-analyse.

Tout d'abord, la question de l'origine et de la nature de la perception du traducteur. Le problème est de savoir si cette perception est elle-même l'intuition originaire de la traduction ou bien si elle est dérivée d'une origine à déterminer.

Dans le premier cas, elle serait à la base de tout savoir-faire du traducteur et il faudrait la «travailler» pour ainsi dire, afin de cultiver et d'améliorer le savoir théorique et pratique du traducteur.

Dans le second cas, la perception serait envisagée comme un mode d'appréhension du monde et du texte, mode dérivé de la sensation personnelle ou bien 
des informations dont disposerait le traducteur à un moment donné au sujet d'un événement particulier. Si c'est le cas, il est important d'enrichir les connaissances du traducteur sur toutes les thématiques touchant à son travail pour assurer la plus grande fiabilité du produit final (le texte-traduit).

La nature de la perception traductionnelle est ainsi une question de fond qui conditionne aussi bien l'exercice de la profession que la formation à la traduction. Cette perception ne nous met pas seulement en présence d'un texte différent, mais aussi en présence d'un sens particulier qu'a ce texte pour le traducteur et, par transitivité, pour nous comme récepteurs finaux.

Nous insistons ici sur le fait qu'il s'agit d'un «sens» particulier et non pas d'une "interprétation», car l'interprétation est une activité intellectuelle qui est elle-même soumise à la perception du traducteur-interprétant. Elle est secondaire par rapport à la perception, dérivée de ce que pense ou croit le traducteur au sujet des mots et des idées d'un texte ou d'un auteur donné.

Cette distinction entre interprétation et perception vise à préciser que le traducteur n'est jamais indifférent face à l'objet qu'il traduit: celui-ci est perçu comme facile ou difficile, général ou spécialisé, léger ou profond, intéressant ou ennuyeux, etc.

Même dans les cas où le donné-à-traduire serait perçu avec indifférence, il n'est pas pour autant pourvu d'une signification donnée objectivement par le traducteur dans son état même d'indifférence.

Ainsi, le sens du texte-traduit est presque toujours le produit d'un acte perceptif chargé d'émotivité ou d'affectivité au sens philosophique du terme. Ces deux états psychologiques impliquent un travail de sélection concernant la manière dont le texte (ou le donné-à-traduire) est perçu. En bref, la neutralité du traducteur est un leurre et les enjeux de la traduction sont avant tout éthiques et déontologiques.

\section{L' analyse est nécessaire}

Nous avons choisi de parler du traducteur dans son rapport à la perception, mais nous pouvons évoquer la même problématique en ce qui concerne l'interprète, en suivant la distinction philosophique entre la perception sensorielle fondée sur le corps et la perception intellectuelle fondée sur l'esprit.

Dans le travail de l'interprète, la perception de manières d'être et d'états émotionnels (l'agressivité, la joie, la spontanéité, etc.) influe sur la façon de traduire les paroles de l'autre. De même, la perception des affects et des idées est un facteur déterminant dans le processus d'interprétation. Pouvoir distinguer ces différents états permet de voir en quoi la perception englobe et transcende le travail intellec- 
tuel de l'interprétation classique. Percevoir chez autrui de la déception, percevoir à ses gestes ou à ses paroles de la contrariété, influe souvent sur ce qu'il veut dire et sur ce que l'interprète peut transmettre ou traduire.

Percevoir ces signes de la communication non verbale signifie qu'il faut à chaque fois les décoder et les lire d'une certaine manière. L'interprète voit un froncement de sourcils, observe les gesticulations du locuteur, mais il doit les relier à l'énoncé prononcé et traduire le propos en conséquence. C'est le propre de la perception interprétative car les sens de l'interprète sont sollicités et non pas seulement sa connaissance ou sa compétence linguistique. S’il perçoit dans le froncement de sourcils une contrariété ou de la colère dans les gesticulations, autrement dit s'il perçoit la signification de ces mouvements corporels, cela le conduira forcément à produire une interprétation qui rend compte de sens moins manifestes et plus subjectifs.

Ces exemples montrent que l'acte de percevoir exige un effort personnel puisque l'interprète tente, grâce à son expérience et à sa compétence, de saisir à travers un signe du corps (un geste, un rictus), le sens véritable d'une expression linguistique ou l'implicite d'une idée ou encore la portée d'une intention de communication. La perception enveloppe le travail intellectuel de l'interprétation en en construisant l'objet et en s'adaptant aux données.

Mais contrairement au mot «traduction», celui d' «interprétation» est porteur d'ambiguïté. Plus fortement que la traduction, l'interprétation nomme l'expérience corporelle de recevoir et de transmettre les paroles d'autrui à travers les organes des sens. Si l'on veut absolument distinguer nettement «traduction» et «interprétation», il faut insister sur le fait que cette dernière se définit comme une réception, en son propre corps, d'une parole étrangère, alors que la traduction consiste à projeter une parole intérieure sur un objet existant à l'extérieur de soi, le $\operatorname{corp}(u) s$ textuel.

Si nous distinguons les deux termes, traduction et interprétation, ce n'est pas tant pour les opposer mais avant tout pour insister sur le fait que nous percevons différemment les choses quand on traduit et quand on interprète. Cette différence de perception est tributaire au moins du facteur corporel et temporel qui implique une instantanéité et une rapidité de l'acte perceptif chez l'interprète, tandis que le traducteur peut se permettre de prendre - comparativement - le temps de la réflexion et de l'analyse.

Dans un cas, l'interprète réagit à ce qui est dit, tandis que dans l'autre, le traducteur agit sur le texte. D'où la définition, d'un point de vue temporel et perceptif, de la traduction comme un mode d'activité et de l'interprétation comme un art de la réactivité. 


\section{Le traducteur n'est pas un homme invisible}

Face au monde, l'interprète-traducteur se pose donc comme un réacteur percevant. La distinction stricte entre ce réacteur-percevant et le donné-à-traduire est difficilement défendable. L'acte perceptif de l'interprète-traducteur a lieu dans la communication et non pas en soi. Autrement dit, la traduction-interprétation n'est pas celle d'un sujet qui surplombe le message, de même que le monde perçu n'est pas un pur objet reposant en lui-même indépendamment du sujet percevant.

Mieux, la communication n'existe pour moi qu'à travers une perception qui la valorise ou la dévalorise, qu'à travers un intérêt et une émotivité qui colorent la perception que j'en ai. C'est pourquoi cette évaluation des choses perçues dans la communication ne peut se comprendre comme relevant d'un jugement froid et détaché du traducteur. Cette évaluation tient à notre relation intime avec le communiqué.

Cela est d'autant plus vrai que les communications ne sont pas des objets neutres auxquels on attribuerait ensuite, par un jugement extérieur à eux, une qualification qui les ferait alors exister pour nous d'une certaine manière. Les communications sont au contraire vécues et non pas seulement jugées: elles sont vécues comme valeur. C'est dire que la traduction analytique n'est pas une objectivation de la communication et qu'elle relève bien plutôt d'une sorte de «foi»: elle coexiste avec l'original dans une forme d'évidence, à travers notre désir d'exister en elle et par elle.

Pour comprendre la véritable nature de l'acte traductionnel, il nous faut séparer d'emblée les mots à traduire du sens perçu. La traduction est à la fois un acte mental et affectif: certes, c'est l'esprit qui réfléchit lors de la traduction, mais on traduit aussi avec ses tripes. Traduire engage une puissance d'agir et, simultanément, toute la puissance de penser. L'esprit ne peut percevoir que si le sujet est affecté, faisant de la traduction une simple orientation de l'esprit à un moment donné et suivant une perception particulière. La compréhension des messages à traduire indique plus l'état de notre perception que le sens exact des messages. C'est ce qui explique en grande partie le phénomène récurrent des retraductions, sans cesse renouvelées.

Il faut faire une distinction, dans la communication multilingue, entre la dimension représentative de la traduction et sa dimension indicative. Une traduction représente un message comme nous étant présent parce qu'elle est l'image de notre compréhension. Traduire le sens c'est davantage l'imaginer à partir de ce que nous sommes qu'à travers ce qu'il est réellement.

Mais en même temps qu'une traduction nous représente le message original, elle indique l'état de notre cognition, c'est-à-dire la manière dont nous appréhen- 
dons les objets du monde. C'est donc le même acte traductionnel qui est représentatif de l'original et indicatif de notre propre perception.

Si toute traduction est une perception indicative d'un état intérieur (notre perception) et représentative d'un objet extérieur (le donné-à-traduire), il est clair qu'il ne peut y avoir de traduction neutre, objective, non orientée. En toute rigueur, il ne peut même pas y avoir de traduction sans affect. Le traducteur ne peut être déconnecté du monde et l'esprit ne peut aborder les messages sans aucune perception préalable. Cela est d'autant plus vrai qu'une traduction n'existe jamais seule puisque chaque message, chaque texte, se définit sur le fond d'une mémoire entendue comme une intertextualité. Chaque traduction actuelle rappelle toutes les traductions passées et se trouve tendue vers des traductions à venir, qui tissent ensemble le vécu mnémonique de tout traducteur.

\section{Le cogito pré-traductionnel}

La plupart du temps, nous traduisons des genres de textes connus. Que nous traduisions des romans, des essais, des publicités ou des manuels de l'utilisateur, nous empruntons des chemins que nous avons déjà pratiqués (narratifs, argumentatifs, informatifs, etc.) et nous utilisons des procédés déjà connus (l'emprunt, le calque, l'adaptation, la transposition, etc.). Mais notre traduction ne se réduit pas à une actualisation de nos souvenirs intertextuels ni de nos perceptions passées.

La traduction-analyse s'attache à décrire les modalités spécifiques selon lesquelles l'interprète-traducteur aborde un objet-à-traduire, l'imagine, le conçoit, le juge, le ressent, le traite, etc. La réflexion du traducteur désigne le retour sur sa propre expérience dans un double regard d'observation et d'évaluation (l'autocritique). Car la traduction est certes dirigée sur un objet, mais le traducteur peut à tout moment se retourner sur lui-même et non plus sur cet objet extérieur, pour réfléchir sur sa pratique. Ainsi, tandis qu'il traduit, il peut considérer intérieurement son acte de traduction et constater qu'il le fait distraitement ou non, avec application, ennui, engouement, etc.

Le philosophe Merleau-Ponty fait de cette «réflexivité» le cœur de la relation qu'entretient le sujet avec le monde. Penser la traduction à partir de cet horizon philosophique, c'est affirmer la communauté de destin de celui qui traduit avec l'auteur de ce qui est traduit, c'est affirmer la nécessité pour celui qui traduit d'exister au moment même où il fait exister le texte-traduit: "Celui qui voit ne peut posséder le visible que s'il en est possédé» (Merleau-Ponty 1964: 177).

Il faut insister ici sur le fait que la traduction, en tant qu'acte perceptif, ne saurait être comprise comme une représentation distanciée du texte, sous prétexte que le sujet traducteur serait foncièrement séparé de l'objet traduit. Même si l'on 
use du vocabulaire de la «représentation" pour des raisons didactiques, on ne peut plus concevoir la traduction dans l'opposition entre un sujet (celui qui reçoit la commande du client) et un objet (ce qui serait le support substantiel du travail). La traduction est plutôt un trait d'union entre mon être et les autres êtres, exprimant l'établissement d'une relation dynamique entre êtres à un moment donné de l'histoire humaine.

Reste la «distance», inhérente à l'acte traductionnel. Certes, traduire c'est à la fois se rendre capable d'approcher le texte-à-traduire (ici la traduction tend à réduire la distance). Mais par la même, le traducteur tend à augmenter cette distance en transférant le sens perçu dans une autre langue. Cette distance reflète son aptitude simultanée à «affecter» le monde et à «être affecté» par lui, selon les termes de Merleau-Ponty.

Signalons au passage qu'il faut envisager un renoncement progressif à l'égard de la dichotomie longtemps intériorisée entre la «source» (texte de départ) et la «cible» (texte d'arrivée). Il s'avère nécessaire aujourd'hui de replacer le traducteur, en tant que sujet percevant, au centre du débat sur les tenants et les aboutissants de la traduction. Dès lors se brouille la frontière de la passivité et de l'activité, ou plutôt de la réception et de la production du sens. Une image forte permet de saisir ce changement de perspective.

\section{Le cube traductologique}

Il est insuffisant de caractériser le texte-à-traduire seulement à partir de son «sens » ou de son «contenu». La portée de la traduction ne consiste pas en un pôle objectif préexistant à la tension qui s'instaure avec l'entrée en scène du traducteur. Le traducteur fait autant exister le sens du texte que le sens ne le fait exister. Plus précisément: si le sens perçu n'existe que par un traducteur qui le perçoit et lui donne une valeur, de la même manière ce sujet percevant ne peut être perçu lui-même que dans son mouvement de traduction.

L'acte de traduire est l'enjeu central de la réflexion parce qu'il fait exister simultanément le sens pour le traducteur et le traducteur dans le monde. En se débarrassant de l'idée d'une primauté du sens dans l'acte de traduire, on peut davantage mettre en valeur l'existence de la traduction en elle-même. Mais comment saisir le propre de la traduction? Les travaux du philosophe Jean-Paul Sartre sur l'imagination créatrice permettent d'entrevoir une réponse à cette question.

On doit apprendre les objets c'est-à-dire multiplier sur eux les points de vue possibles. Lobjet lui-même est la synthèse de toutes ces apparitions. . Qu'est-ce que cela signifie pour nous? La nécessité de faire le tour des objets (Sartre 1940: 22). 
Cela appelle une ouverture du traducteur à un savoir qu'il n'a pas encore, c'est la nécessaire exploration de l'objet par étapes et progressivement. L'exemple du «cube» décrit par Sartre permet de saisir la complexité du phénomène d'appréhension du sens dans la traduction:

L'objet, quoi qu'il entre tout entier dans ma perception, ne m'est jamais donné que d'un côté à la fois. On connaît l'exemple du cube: je ne puis savoir que c'est un cube tant que je n'ai pas appréhendé ses six faces; je puis à la rigueur en voir trois à la fois, mais jamais plus. Il faut donc que je les appréhende successivement (Sartre 1940: 21).

Dans la traduction, le texte-à-traduire est généralement un objet présent devant moi comme objet à connaître. Certes, il peut être un texte électronique mais cela n'enlève rien à son caractère sensible, concret, d'objet-à-traduire. Le problème n'est donc pas dans l'objet mais dans la manière de l'appréhender. Nous proposons une représentation de l'objet traductionnel suivant l'image du cube et la conception réflexive évoquée précédemment (Figure 1). L'image du «cube traductologique» permet de saisir la complexité du processus de traduction. Le cube possède, en effet, six faces mais il est possible d'en voir seulement trois à la fois. Les faces accessibles au traducteur sont celles de ses propres conceptions, perceptions et intentions. Mais il doit nécessairement retourner le «cube» pour voir les conceptions, les perceptions et les intentions des «autres», ceux qui parlent à travers le texte source.

Cette image familière et dynamique permet de comprendre que le processus de traduction correspond fondamentalement à une activité réflexive en perpétuel ajustement. C'est pourquoi, elle ouvre des champs nouveaux d'exploitation didactique. La méthodologie de base consisterait à enseigner le processus de traduction suivant trois étapes essentielles: déconstruction du cube traductologique, choix des facettes pertinentes pour la traduction, reconstruction du cube par le traducteur.

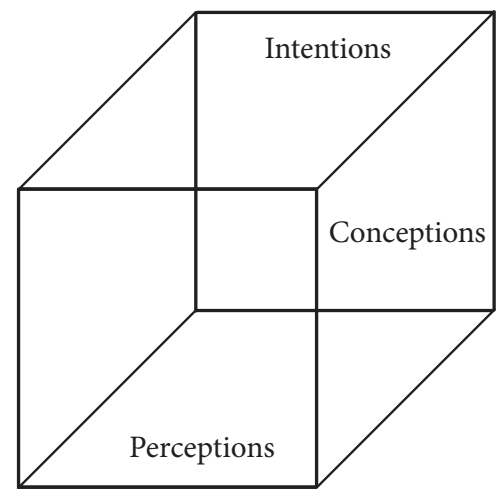

Figure 1. Le cube traductologique (Perceptions, Conceptions, Intentions) 
La difficulté réside dans le fait que l'on doit reconnaitre de manière explicite qu'il n'y a pas une seule manière d'analyser les mots: ce que l'on obtient comme analyse est un objet construit que l'on caractérise par les conditions qui ont présidé à sa construction (Kamp et Reyle 1993).

Car le langage humain présente certaines spécificités dont il faut tenir compte. Tout d'abord, la capacité à exprimer l'intention et l'avenir et non seulement le réel et le présent. C'est la condition sine qua non de la capacité d'abstraction. Ensuite, la capacité à exprimer des liens logiques: le langage permet que se développent un raisonnement et une argumentation au sujet d'un phénomène ou d'une situation. Enfin, la capacité à exprimer la mémoire du passé: l'aboutissement le plus achevé de cette capacité est la transmission de l'expérience par divers moyens (écriture, audio, vidéo, etc.).

En conséquence, on peut distinguer trois grands niveaux d'analyse:

- Le premier niveau est celui du langage comme système de signification: par exemple, le mot "khadim» en arabe signifie "serviteur» (sens premier, dénoté);

- Le deuxième niveau est celui du langage comme système de connaissance: par exemple, le même mot «khadim» est employé comme titre officiel du Roi d'Arabie Saoudite (khadim al-Haramayn, Serviteur des Lieux Saints).

- Le troisième niveau est celui du langage comme système de communication: par exemple, ce même mot «khadim» est employé dans le proverbe arabe «khadimu al-qawmi sayyiduhum» (Le serviteur des gens est leur maître) qui renverse totalement le sens initial du mot puisque le «serviteur» au niveau de la signification devient «seigneur» au niveau de la communication.

Du point de vue cognitif, ces trois niveaux de perception du langage se confondent et se complètent pour produire le sens global du message, à un moment donné et dans une situation particulière. Mais tout le monde ne peut pas accéder simultanément à ces différents niveaux, car cela suppose une maîtrise suffisante de la complexité du système.

Le «cube traductologique» permet de mieux comprendre le lien qui unit le monde subjectif des traducteurs aux données objectives du texte-à-traduire. En effet, les interprètes-traducteurs associent inconsciemment ce qu'ils ressentent à la cause de leur impression; ils ressentent une certaine quantité d'émotions, définie par contraste à la situation qu'ils vivent, et ils cherchent inconsciemment à lui attribuer une grandeur pour pouvoir traduire. Ce faisant, ils objectivent une donnée textuelle ou discursive qui appartient en toute rigueur à la conscience subjective.

Nos recherches sur la cognition de la traduction, illustrées métaphoriquement par l'image du cube, montrent qu'il existe trois grandes phases logiques et chronologiques dans l'appréhension du sens. 
La première phase est celle de la perception: elle concerne l'effet des mots du texte-à-traduire sur les facultés du sujet traduisant. Cet effet est un mouvement qui va des mots jusqu'au cerveau en passant par divers centres nerveux et mémoriels. Le mécanisme de la compréhension du texte-à-traduire possède une dimension perceptive dans laquelle les composants de l'objet traductionnel interagissent avec les sens mémorisés par le traducteur ou percepts (Deleuze 1981).

La deuxième phase est celle de la conception: elle concerne l'union entre les concepts issus du texte et les perceptions emmagasinées dans la mémoire du sujet traduisant. Il y a là une compréhension par l'esprit des mouvements du texte en référence aux acquis antérieurs du traducteur. Les impressions senties ne correspondent pas nécessairement aux objets à traduire, mais elles permettent le déclenchement du processus traductionnel. La conjonction des significations mentales et textuelles autorise le déchiffrement et l'interprétation du texte.

La troisième phase est celle de l'intention: elle concerne la mise en forme de la combinaison de "percepts» et de "concepts» issue des deux phases précédentes suivant une visée particulière, qu'elle soit de nature personnelle, objective ou institutionnelle. L'activité d'équivalence à proprement parler est le moment clé de cette phase intentionnelle parce qu'elle correspond précisément à l'assignation d'un sens téléologique, général ou particulier, observable au niveau macro ou micro textuel.

Ainsi, la traduction apparait en définitive comme une dynamique entre un mouvement d'essence passive (la perception), auquel succède un mouvement d'essence active (la conception), tendu vers un objectif précis (l'intention) qui détermine les potentialités de mise en forme finale de la communication (Guidère 2008).

\section{Mise en perspective}

La traduction est «orientée » parce qu'il existera toujours des conceptions, des perceptions et des intentions différentes selon les textes et selon les traducteurs. C'est cette orientation de l'esprit qu'il convient d'analyser en détail et de façon réflexive.

La théorie analytique de la traduction s'intéresse au processus de traduction. Elle récuse la dichotomie «texte source» versus «texte cible». Pour nous, le "sens» ne se trouve ni dans l'un ni dans l'autre; il est dans le médium que représente le traducteur à un moment donné. Affirmer que le sens est perceptif signifie qu'il est tributaire de la compréhension du sujet traduisant. En d'autres termes, le traducteur doit réfléchir sur son activité de traduction afin de limiter les aléas inhérents à cette activité. 
Enfin, la théorie analytique part du principe que la compétence linguistique du traducteur est globalement acquise. Dans un cursus de traduction, ce dernier n'est plus censé apprendre une langue mais une manière de traduire des productions langagières authentiques. Le cours de traduction n'est en aucun cas un cours de langue, et l'apprentissage des langues de travail est un préalable non négociable à l'apprentissage de la traduction. Ce préalable permet d'évacuer les problèmes d'enseignementapprentissage des langues au profit des problématiques propres à la traduction.

\title{
Références
}

Lederer, M., La traduction aujourd'hui - le modèle interprétatif, Paris, Lettres Modernes, 1994, $196 \mathrm{p}$.

Seleskovitch, D. et M. Lederer ( $4^{\mathrm{e}}$ éd.), Interpréter pour traduire, Paris, Didier Érudition, 2001, $311 \mathrm{p}$.

Berman A. 1984, L'Épreuve de l'étranger, Paris, Flammarion, 311 p.

Berman A. 1995, Pour une critique des traductions: John Donne, Paris, Flammarion, 275 p.

Huxley A., Les portes de la perception, trad. J. Castier, Éd. du Rocher, 1954, 171 p.

Guidère M., Introduction à la traductologie, Bruxelles, De Boeck, 2008, 240 p.

Guidère M., Traduction et communication multilingue, Bruxelles, De Boeck, 2008, 271 p.

Guidère M., "Professional Translation and National Security", in Proceedings of the 45th Annual Conference of ATA, October 13-14, Toronto, 2004, pp. 333-43.

Baker M., Translation and Conflict: Mediating Competing Narratives, Routledge, 2006, 208 p.

Merleau-Ponty M., Phénoménologie de la perception, Paris, Gallimard, 1945, 531 p.

Merleau-Ponty, Le visible et l’ invisible, Paris, Gallimard, coll. «Tel», 1964, 364 p.

Meschonnic H. 1973, Pour la poétique II. Épistémologie de l'écriture poétique de la traduction, Paris: Gallimard, $457 \mathrm{p}$.

Meschonnic H. 2007, Éthique et politique du traduire, Lagrasse: Verdier, 185 p.

Sartre J.-P., L' imaginaire, coll. «idées», Paris, Gallimard, 1940, 379 p.

Bergson H., Matière et mémoire, PUF, 1990, 280 p.

Deleuze G., Logique de la sensation, Paris, Seuil, 1981, 158 p.

Changeux J.-P., L'Homme neuronal, Paris, Fayard, 1983, 419 p.

\begin{abstract}
Despite the major changes that occurred in the world during the last decade, translation theory has not taken into account the inevitable impact of these changes on the translation profession. Neither theorists nor professional translators have analyzed enough the remarkable change in perspective and method that occurred in the language field as a whole and primarily in the translation practice. This paper presents some important aspects of this change of perspective which calls for a new theoretical paradigm. The latter, which we refer to as translationanalysis, is currently a fast-growing activity.
\end{abstract}




\section{Résumé}

Malgré les bouleversements majeurs qu'a subis le monde au cours de la dernière décennie, la théorie de la traduction n'a pas pris en considération les conséquences inévitables de ces bouleversements sur le métier de traducteur. Ni les théoriciens ni les praticiens n'ont suffisamment analysé le changement remarquable de perspective et de méthode qui s'est opéré dans le domaine langagier et, en premier lieu, dans celui de la traduction. Cet article présente quelques aspects importants concernant ce changement de perspective, aspects qui militent en faveur d'un nouveau paradigme théorique, la traduction-analyse, qui correspond à une pratique actuellement en pleine expansion.

\section{Biographie}

Mathieu Guidère est professeur à l'Université de Genève (ETI, Suisse). De 2003 à 2007, il a été directeur de recherche au ministère de la Défense français. Spécialiste de veille stratégique multilingue, il a publié plusieurs ouvrages sur la traduction et la propagande, notamment Les Martyrs d'Al-Qaïda (éditions du Temps, 2005), Le Manuel de recrutement d'Al-Qaïda (éditions du Seuil, 2006) et Al-Qaïda à la conquête du Maghreb (éditions du Rocher, 2007). Site web: www. guidere.org.

Addresse: $\mathrm{xxxx} \mathrm{xxxx} \mathrm{xxxxx}$

Courriel: Mathieu.Guidere@eti.unige.ch 